\title{
Impacto da condição corporal sobre a taxa de prenhez de vacas da raça nelore sob regime de pasto em programa de inseminação artificial em tempo fixo (iatf)
}

\section{Impact of body condition on pregnancy rate of cows nellore under pasture in fixed time artificial insemination (tai) program}

\author{
Marcelle Christine Nascimento Ferreira ${ }^{1}$; Rodolfo Miranda ${ }^{2}$; \\ Marcelo Abidu Figueiredo ${ }^{3}$; Orlando Marques Costa ${ }^{3}$; Helcimar Barbosa Palhano ${ }^{3 *}$
}

\section{Resumo}

\begin{abstract}
O objetivo deste trabalho foi avaliar o impacto da condição corporal sobre a taxa de prenhez de vacas Nelore, rebanho comercial, submetidas a programas de inseminação artificial em tempo fixo (IATF). Foram utilizadas 181 vacas multíparas da raça Nelore, na região das baixadas litorâneas do estado do $\mathrm{RJ}$, com mais de cem dias decorridos do parto, mantidas em regime de pasto e divididas em dois grupos submetidos ao mesmo protocolo de sincronização para IATF (D0- 2,0mg de benzoato de estradiol + dispositivo intravaginal bovino com $1,0 \mathrm{~g}$ de progesterona, D8- retirada do implante $+250 \mu \mathrm{g}$ de cloprostenol sódico+ 300 UI de eCG, D9- 1,0mg Bz. Estradiol, D10- IATF). Os grupos foram divididos segundo escore de condição corporal (ECC com escala de 1-5) em: grupo I, $n=96$ : vacas com ECC $\geq$ 3,0 e grupo II, $n=85$ : vacas com $E C C \leq 2,5 \geq 2,0$. Todas as fêmeas foram expostas aos touros, a partir de 24 horas após a IATF para repasse, permanecendo com os mesmos até o fim da estação de monta. A taxa de prenhez total foi de $86,5 \%$ (83:96) e 65,9\% (56:85) para os grupos I e grupo II, respectivamente. Os dados foram avaliados pelo teste do qui-quadrado e os resultados analisados apontam diferença estatisticamente significativa $(\mathrm{P}<0,05)$ entre os grupos, podendo-se concluir que as fêmeas de pior condição corporal ( $\mathrm{ECC} \leq 2,5 \geq 2,0$ ), apresentaram menor taxa de prenhez quando comparadas aquelas de melhor condição (ECC $\geq 3$ ) quando submetidas a programa de IATF.
\end{abstract}

Palavras-chave: Condição corporal, taxa de prenhez, IATF, vacas nelore

\begin{abstract}
The aim of this study was to evaluate the impact of body condition on pregnancy rate of Nellore cows, commercial herd undergoing artificial insemination programs in fixed time (TAI). 181 cows were used multiparous Nellore, the coastal plains region of the state of Rio de Janeiro, with more than one hundred days after birth, kept on pasture and divided into two groups subjected to the same synchronization protocol for TAI (D0-2, $0 \mathrm{mg}$ of estradiol benzoate + device with $1.0 \mathrm{~g}$ bovine intravaginal progesterone implant removal D8-250 $\mathrm{g}$ of cloprostenol + + $300 \mathrm{IU}$ of eCG, D9-Bz $1.0 \mathrm{mg}$. Estradiol, D10-TAI). The groups were divided according to body condition score (BCS with scale of 1-5) in Group I, $n=96$ : BCS $\geq 3,0$, Group II, $n=85$ : BCS $\leq 2.5 \geq 2.0$. All females were exposed to bulls, from 24 hours to pass after
\end{abstract}

\footnotetext{
${ }^{1}$ Discente do Curso de Medicina Veterinária, Universidade Federal Rural do Rio de Janeiro, UFRRJ, Rio de Janeiro, RJ. E-mail: marcellechris22@hotmail.com

${ }^{2}$ Discente do Curso de Zootecnia, UFRRJ, Rio de Janeiro, RJ. E-mail: rodolfosalame@yahoo.com.br

${ }^{3}$ Profs. Adjuntos, Dept ${ }^{\circ}$ Biologia Animal, UFRRJ, Rio de Janeiro, RJ. E-mail: marceloabidu@gmail.com; orlando@ufrrj.br; helcimarpalhano@gmail.com

* Autor para correspondência
} 
TAI, remaining with them until the end of the breeding season. The overall pregnancy rate was $86.5 \%$ (83:96) and 65.9\% (56:85) for group I and group II, respectively. Data were evaluated by chi-square analysis and the results show a statistically significant difference $(\mathrm{P}<0.05)$ between groups, which may be concluded that the females of poor body condition ( $\mathrm{BCS} \geq 2.0 \leq 2.5$ ), have lower pregnancy rates compared to those best condition (BCS $\geq 3$ ) when subjected to TAI program.

Key words: Body condition, pregnancy rate, TAI, Nellore cows

\section{Introdução}

A atual demanda mundial pela produção de carne bovina para consumo humano exige dos produtores um grande esforço para a melhoria dos indicadores de eficiência reprodutiva e produtiva de seus rebanhos, com vistas à melhoria da taxa de desfrute e conseqüente retorno econômico da atividade.

Neste contexto, a inseminação artificial em tempo fixo (IATF) que vem despontando como uma promissora ferramenta de manejo reprodutivo de bovinos de corte pode contribuir de forma significativa para melhoria desses indicadores, contudo, o planejamento nutricional deve proporcionar a manutenção de boas condições corporais para maximização dos resultados a serem obtidos com a IATF.

É conhecido o efeito negativo da mamada sobre o ciclo estral em vacas de corte (MALVEN, 1984; WHISNANT; KISER; THOMPSON, 1986), determinando anestro pós-parto em função da inibição da secreção de GnRH por opióides endógenos (encefalinas, endorfinas e dinorfinas), o que se torna mais grave em fêmeas com deficiências nutricionais (BUSTAMANTE et al., 1997) e o escore de condição corporal (ECC) baixo, dentre outros fatores que influenciam a duração do anestro (BARUSELLI et al., 2002; MARQUES et al., 2003) contribuindo para baixos índices reprodutivos (RIGOLON et al., 2008).

Segundo Valle, Andreotti e Thiago. (1998), vacas com boas condições corporais ao parto retornam ao cio mais cedo e apresentam maiores índices de concepção sendo que a suplementação de vacas nos períodos pré e pós parto resultam em incremento do peso corporal, o que interfere positivamente na taxa de prenhez, uma vez que vacas com melhores condições corporais durante a estação de reprodução apresentam maior probabilidade de emprenhar (ALMEIDA; LOBATO; SCHENKEL, 2002; GODOY et al., 2004, SONOHATA et al., 2009).

Assim, o anestro pós-parto, cuja duração é influenciada pela condição corporal pré e pós-parto, e as falhas na detecção de estro no início da estação de monta em rebanhos comerciais de corte, são fatores que contribuem para o prolongamento do período de serviço (RICHARDS; SPITZER; WARNER, 1986; RUAS et al., 2005; MENEGHETTI; VASCONCELOS, 2008; VASCONCELOS; VILELA; SÁ FILHO, 2009), determinando um baixo desempenho reprodutivo (ALMEIDA; LOBATO; SCHENKEL, 2002) e baixa taxa de desfrute dos mesmos, sendo necessário uma nutrição adequada nestes períodos para que se obtenha uma melhoria na taxa de prenhez (SAMPEDRO; GALLI; VOGEL, 2003).

Ainda no que diz respeito à relação entre nutrição e reprodução, a condição corporal (CC) exerce uma influência direta sobre a fertilidade, podendose manter a ciclicidade em fềmeas com ECC 2 ou mais (escala de 1 a 5), sendo ainda influenciada por outros fatores como a raça e a evolução do peso (SHORT et al., 1990; CUTAIA et al., 2003) e a sua avaliação torna-se de grande importância na tomada de decisão para os produtores (SANTOS et al., 2009).

Ao analisar 6857 IATF realizadas com vacas secas, vacas com cria ao pé e novilhas, mestiças de zebu com raças britânicas, Cutaia et al. (2003) observaram uma taxa de prenhez média de $54,9 \%$ com um mínimo de $28,7 \%$ para vacas com cria ao pé e ECC 2 e um máximo de $75 \%$ para novilhas com 
ECC 3, sugerindo ECC mínimo de 2,5 com ideal de 3 para obtenção de bons resultados em programas de IATF.

Embora a gordura corporal seja apenas um indicador de características de alguns dos mecanismos que controlam o recomeço da atividade estral e, portanto, não é um efeito direto de controle desses mecanismos, seu uso se justifica pela falta de um melhor preditor em nível de campo, além do fato de o ECC ser recomendado como parte do manejo reprodutivo (TESS; KOLSTAD, 2000; FONTOURA JUNIOR et al., 2009) e ser ainda analisado ao parto como o principal fator determinante do intervalo parto - primeiro cio e taxa de prenhez em vacas multíparas, além de ser um confiável indicador do desempenho reprodutivo pós-parto em primíparas (WETTEMANN; BOSSIS, 1999; MORRISON; SPITZER; PERKINS, 1999; DEROUEN et al., 1994; LAKE et al., 2005; FONTOURA JUNIOR et al., 2009).

Em sistema de monta natural, McManus et al. (2002) avaliaram os diversos fatores que influenciaram o intervalo de partos (IP), o índice de fertilidade real $(\mathrm{FR}=$ peso bruto à desmama $\mathrm{x}$ $365 /$ dias para parir), razão do peso do bezerro ao nascer (RPN) e razão de peso ao desmame (RPD) em relação ao peso da vaca ao parto e a desmama no rebanho de gado mestiço da Embrapa Cerrados. Foram analisados dados de 4469 parições, coletados no período entre 1976 e 1999 e os resultados apontaram para a influência positiva da condição corporal da vaca ao parto sobre a FR e da condição corporal da vaca ao desmame sobre o IP e sobre a FR, considerando importante a influência da condição corporal sobre fatores produtivos no rebanho estudado, mostrando a necessidade do controle do peso das matrizes para a melhoria dos índices produtivos e reprodutivos.

Vieira et al. (2005) avaliaram durante quatro estações de monta, o efeito da ordem do parto e da condição corporal (ECC de 1 a 5) sobre o desempenho reprodutivo de 468 fêmeas nelore, concluindo que a ordem do parto influenciou a taxa de prenhez (melhores resultados entre o $3^{\circ}$ e o $8^{\circ}$ parto) e a manutenção da condição corporal acima de 3,0 foi condição necessária para a boa eficiência reprodutiva das vacas na fase de cria.

Da mesma forma Cutaia e Bó (2004) verificaram correlação de $90 \%$ do efeito da CC no momento da IATF sobre a taxa de concepção em vacas submetidas a protocolos hormonais, obtendo-se maiores taxas de concepção em programas de IATF com aquelas fêmeas de melhor CC (LAMB et al., 2001; MENEGHETTI; LOSI; MARTINS, 2005; MENEGHETTI ; VASCONCELOS, 2008).

O objetivo deste trabalho foi avaliar o impacto da condição nutricional, por meio da avaliação do ECC, sobre a taxa de prenhez de vacas da raça nelore sob regime de pasto em programa de IATF.

\section{Material e Métodos}

Entre os meses de novembro de 2009 e maio de 2010, foram avaliadas 181 fêmeas multíparas e lactantes da raça nelore com idade compreendida entre 5 e 15 anos e com mais de cem dias decorridos do parto, de um rebanho comercial com aproximadamente 1500 matrizes, localizado no município de Casimiro de Abreu, Região dos Lagos do Estado do Rio de Janeiro, Brasil. Todos os animais foram mantidos em regime de pasto, Brachiaria brizantha cv. Marandú, em sistema extensivo com rotação estacional de piquetes (ocupação período das águas, compreendido entre outubro e março e período seco, entre abril e setembro) formados sob orientação técnica, suplementação de sal mineral e livre acesso à água durante todo o ano.

Com relação ao manejo sanitário da propriedade, todos os animais foram vacinados contra a febre aftosa seguindo o calendário estabelecido pelo Ministério da Agricultura Pecuária e Abastecimento (MAPA). Todas as fêmeas com idade compreendida entre três e oito meses foram vacinadas contra brucelose com a vacina da cepa B-19. Todas as fêmeas em idade reprodutiva foram vacinadas contra 
leptospirose. A vacinação contra clostridioses foi realizada em todos os animais do rebanho uma vez ao ano e nos animais com idade entre três e cinco meses o reforço foi programado para os 30 dias após a primeira vacinação. Os exames de brucelose e tuberculose foram realizados uma vez ao ano.

Como rotina de manejo reprodutivo, a propriedade adotou a inseminação artificial (IA) convencional com sêmen da raça Nelore e das raças Simental e Red Angus, como IA de repasse, utilizando ainda, touros da raça nelore puros de origem (PO) como monta natural de repasse após a segunda IA, permanecendo todas as fêmeas em reprodução com os mesmos até o final da estação de monta que foi realizada entre os meses de novembro a fevereiro.

Para o presente experimento, todas as fêmeas foram submetidas à avaliação visual da condição corporal (CC) sendo classificadas, no início do estudo (novembro de 2009), pelo escore de condição corporal (ECC) que variou de 1 a 5 (1 muito magra e 5 obesa) sendo submetidas também, à triagem ginecológica por palpação retal para avaliar possíveis afecções adquiridas ou congênitas do aparelho genital que pudessem interferir com a inseminação artificial, sendo excluídas aquelas consideradas inaptas. A avaliação por palpação retal foi igualmente utilizada para a identificação de estruturas relacionadas à atividade ovariana, como folículo (FL), corpo lúteo (CL) ou ausência de ambas (L), sendo consideradas com atividade ovariana luteal e cíclica (AOLC) aquelas que apresentaram CL ao exame retal, realizado duas vezes com intervalo de dez dias antes do protocolo de sincronização.

Foram excluídas do experimento fêmeas que eventualmente estivessem gestantes e fêmeas detectadas em cio no momento do exame, sendo as mesmas imediatamente inseminadas.

Como critério de estratificação dos grupos a serem avaliados utilizou-se o ECC, sendo incluídas as fêmeas com escore $\geq 3,0$ no grupo $\mathrm{I}, \mathrm{n}=96 \mathrm{e}$ fêmeas com escore $\leq 2,5 \geq 2,0$ no grupo II, $n=85$. Ambos os grupos foram submetidos ao mesmo protocolo de sincronização para IATF conforme representado na Figura 1 (D0- 2,0mg de benzoato de estradiol + dispositivo intravaginal bovino com $1,0 \mathrm{~g}$ de progesterona, D8- retirada do implante + $250 \mu \mathrm{g}$ de cloprostenol sódico +300 UI de eCG, D9- 1,0mg Bz. Estradiol, D10- IATF). Para a IATF utilizou-se sêmen de um mesmo touro da raça Red Angus para ambos os grupos sendo realizada pelo mesmo inseminador. Os protocolos iniciaram na primeira quinzena do mês de dezembro.

Logo após a IATF, todas as fêmeas foram expostas aos touros de repasse da raça Nelore (PO), sendo mantidas com os mesmos até o final da estação de monta. Os touros utilizados não foram submetidos previamente a exames andrológicosanitários.

O diagnóstico de gestação foi realizado pelo método de palpação retal, 60 dias após o término da estação de monta, contabilizando-se a taxa total de prenhez (IATF + Touro repasse).

Figura 1. Protocolo para sincronização da ovulação utilizado

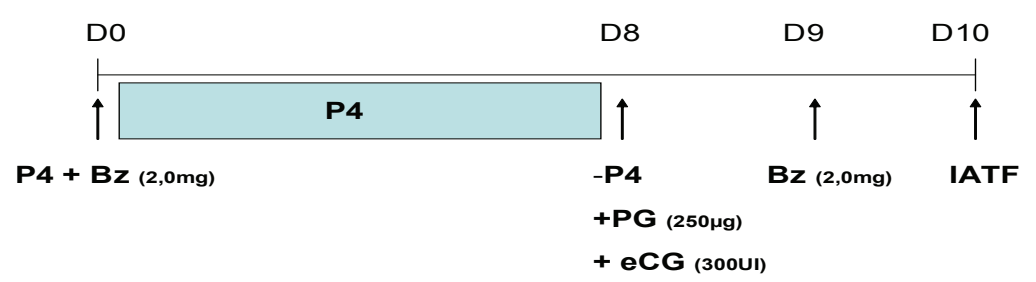

Fonte: Elaboração dos autores. 
Análise estatística foi realizada utilizando-se o teste do Qui-quadrado, ao nível de significância de 5\%, utilizando-se o software Graphpad Prism 5. As taxas de gestação e ciclicidade foram expressas em freqüência absoluta e percentual simples.

\section{Resultados e Discussão}

Para avaliar o efeito da nutrição sobre a taxa de prenhez de vacas da raça Nelore submetidas a programas de IATF, a avaliação da CC por meio de ECC, foi adotada neste trabalho por ser uma medida amplamente utilizada na rotina do manejo reprodutivo de bovinos e ser de fácil mensuração para coleta de dados.

Os resultados obtidos neste experimento apontam para uma influência da nutrição sobre o desempenho reprodutivo de vacas de corte conforme relatado por diversos trabalhos de pesquisa. Assim, vacas de maior ECC apresentaram maior taxa de prenhez, tendo sido de $86,5 \%$ (83:96) e 65,9\% (56:85) para os grupos I e grupo II (Tabela 1), respectivamente, o que pode estar relacionado a um acréscimo na taxa de sincronização para as fêmeas de maior CC, conforme observado por Meneghetti e Vasconcelos (2008) em experimento realizado com vacas primíparas da raça Nelore, confirmando o efeito da nutrição sobre o desempenho reprodutivo.

Tabela 1. Taxa de prenhez de vacas lactantes da raça nelore submetidas a protocolo de IATF com repasse de touro associada ao escore de condição corporal.

\begin{tabular}{lccc}
\hline $\begin{array}{l}\text { Escore de Condição } \\
\text { Corporal (ECC) }\end{array}$ & $\begin{array}{c}\text { ECC } \geq 3 \leq 4 \\
\text { (grupo I) }\end{array}$ & $\begin{array}{c}\text { ECC } \geq 2,0 \leq 2,5 \\
\text { (grupo II) }\end{array}$ & Total \\
\hline $\begin{array}{l}\text { Tx.prenhez }(\%) \\
\text { (IATF+TOURO) }\end{array}$ & $86,5(83: 96)$ & $65,9(56: 85)$ & $76,8(139: 181)$ \\
\hline $\mathrm{N}^{\circ}$ de animais & 96 & 85 & 181 \\
\hline
\end{tabular}

Fonte: Elaboração dos autores.

Os dados foram avaliados pelo teste do quiquadrado e os resultados analisados apontam diferença estatisticamente significativa $(\mathrm{P}<0,05)$ entre os grupos, podendo-se concluir que as fêmeas de pior condição corporal (ECC $\geq 2,0 \leq$ $2,5)$, apresentam menor taxa de prenhez quando comparadas aquelas de melhor condição (ECC $\geq 3 \leq 4$ ) quando submetidas a programa de IATF, conforme reportado por Cutaia et al. (2003) e observado por e Cutaia e Bó (2004) que verificaram correlação de $90 \%$ do efeito da CC no momento da IATF sobre a taxa de concepção em vacas submetidas a protocolos hormonais, correlação também observada em outros trabalhos de pesquisa que relatam maiores taxas de concepção em programas de IATF para aquelas fêmeas de melhor CC (LAMB et al., 2001; MENEGHETTI; LOSI; MARTINS, 2005; MENEGHETTI; VASCONCELOS 2008).

Fatores como a influência da presença do bezerro lactante, a demanda de energia para produção de leite e o cuidado com a cria, associados ao baixo ECC das vacas do grupo II, podem ter impactado negativamente sobre o seu desempenho reprodutivo, conforme observado por Pfeifer et al. (2007), Brauner et al. (2008) e neste trabalho (Tabela 2), observando-se diferença estatisticamente significativa entre os grupos $(\mathrm{P}<0,05)$, apresentando maior taxa de ciclicidade as fêmeas com melhor ECC conforme demonstrado no Figura 2. 
Figura 2. Txs. de ciclicidade e prenhez nos grupos I e II.

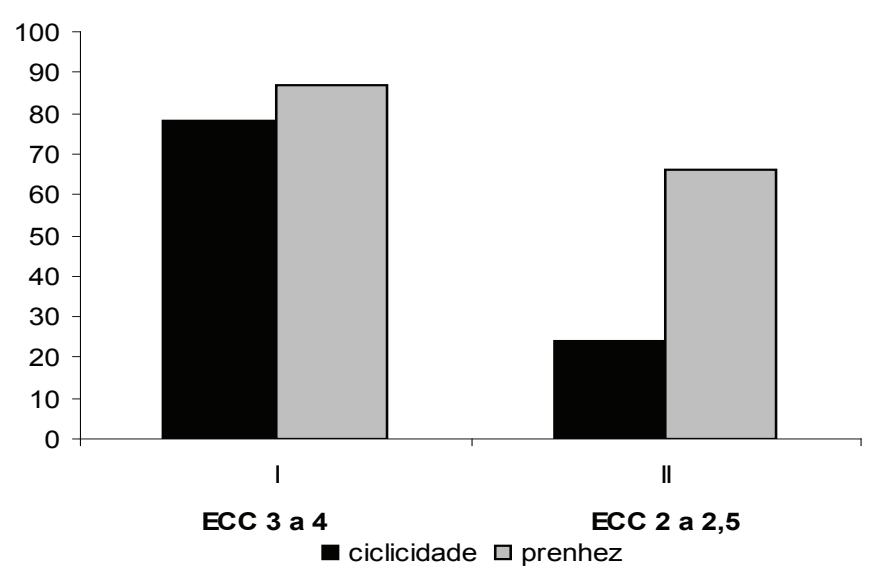

Fonte: Elaboração dos autores.

Tabela 2. Taxa de ciclicidade pré-protocolo de vacas lactantes da raça nelore, avaliadas pela palpação retal, associada ao escore de condição corporal.

\begin{tabular}{lccc}
\hline $\begin{array}{l}\text { Escore de Condição } \\
\text { Corporal (ECC) }\end{array}$ & $\begin{array}{c}\text { ECC } \geq 3 \leq 4 \\
\text { (grupo I) }\end{array}$ & $\begin{array}{c}\text { ECC } \geq 2,0 \leq 2,5 \\
\text { (grupo II) }\end{array}$ & Total \\
\hline $\begin{array}{l}\text { Tx.ciclicidade(\%) } \\
\text { pré-protocolo }\end{array}$ & $78,1(75: 96)$ & $23,5(20: 85)$ & $52,5(95: 181)$ \\
\hline $\mathrm{N}^{\circ}$ de animais & 96 & 85 & 181 \\
\hline
\end{tabular}

Fonte: Elaboração dos autores.

O grau de balanço energético negativo (BEN) em que o animal se encontra é um fator que pode influenciar o intervalo entre $o$ tratamento com progesterona + estradiol e o início da nova onda folicular, determinando menor taxa de sincronização (MENEGHETTI; VASCONCELOS, 2008), o que pode interferir negativamente nos resultados da IATF conforme observado nesse experimento para as vacas do grupo II e segundo Sonohata et al. (2009), a inferioridade do desempenho reprodutivo e a pior condição corporal das vacas lactantes estão associadas a deficiências nutricionais, sendo necessário a mobilização de nutrientes da reserva corporal no período inicial de lactação para suprir a demanda de energia de mantença e de produção não atendidas pela dieta.

Apesar de não ter sido avaliado a influência da ordem do parto sobre a taxa de prenhez, deve-se levar em consideração essa possibilidade conforme apontam Vieira et al. (2005).

\section{Conclusões}

Os resultados obtidos neste estudo permitem concluir que a condição corporal pode afetar o desempenho reprodutivo de vacas Nelore, diminuindo a taxa de concepção no programa de IATF utilizado, observando-se menor desempenho reprodutivo para as fêmeas de pior CC.

Permitem concluir ainda, que a CC também influencia a $\mathrm{AOLC}$, observando-se maior número de vacas cíclicas dentre aquelas que se encontram em melhores condições corporais. Assim o uso do ECC na avaliação nutricional das matrizes, permite a elaboração de estratégias alimentares para as matrizes e suas crias, podendo proporcionar melhores indicadores de eficiência reprodutiva em programas de IATF. 


\section{Referências}

ALMEIDA, L. S. P.; LOBATO, J .F. P.; SCHENKEL, F. $\mathrm{S}$. Data de desmame e desempenho reprodutivo de vacas de corte. Revista Brasileira de Zootecnia, Viçosa, v. 31, n. 3, p. 1223-1229, 2002.

BARUSELLI, P. S.; MARQUES, M. O.; CARVALHO, N. A. T.; MADUREIRA, E. H.; CAMPOS FILHO, E. P. Efeito de diferentes protocolos de inseminação artificial em tempo fixo na eficiência reprodutiva de vacas de corte lactantes Revista Brasileira de Reprodução Animal, Belo Horizonte, v. 26, n. 3, p. 218-221, 2002.

BRAUNER, C. C.; PIMENTEL, M. A.; LEMES, J. S.; PIMENTEL, C. A.; MORAES, J. C. F. Reprodução de vacas de corte em lactação e solteiras submetidas à indução/sincronização de estro. Ciência Rural, Santa Maria, v. 38, n. 4, p. 1067-1072, 2008.

BUSTAMANTE, J. R. B.; FONSECA, F. A.; FONTES, C. A. A.; ESPESCHIT, C. V. Efeito da condição corporal ao parto e da amamentação na eficiência reprodutiva de vacas da raça Nelore. Revista Brasileira de Zootecnia, Viçosa, v. 26, n. 6, p. 1090-1095, 1997.

CUTAIA, L.; BÓ, G. A. Factores que afectan los resultados en programas de inseminación artificial a tiempo fijo en rodeos de cría utilizando dispositivos com progesterona. In: SIMPOSIO INTERNACIONAL DE REPRODUCCIÓN BOVINA, 1., 2004, Barquisimeto. Anales... Barquisimeto: [s.n], 2004. p. 109-123.

CUTAIA, L.; VENERANDA, G.; TRÍBULO, R.; BARUSElli, P. S.; BÓ, G. A. Programas de inseminación artificial a tiempo fijo en rodeos de cría: factores que lo afectan y resultados productivos. In: SIMPOSIO INTERNACIONAL DE REPRODUCCIÓN ANIMAL, 5., 2003, Córdoba, Argentina. Anales.. Córdoba, Argentina: [s.n], p. 119-132,

DEROUEN, S. M.; FRANKE, D. E.; MORRISON, D. G.; WYATT, W. E.; COOMBS, D. F.; WHITE, T. W.; HUMES, P. E.; GREENE, B. B. Prepartum body condition and weight influences on reproductive performance of first-calf beef cows. Journal of Animal Science, Champaign, v. 72, n. 5, p. 1119-1125, 1994.

FONTOURA JÚNIOR, J. A. S.; SIEWERDT, F.; DiONELlO, N. J. L.; CORRÊA, M. N. Modelo de simulação do desempenho reprodutivo de fêmeas bovinas de corte com base no escore de condição corporal. Revista Brasileira de Zootecnia, Viçosa, v. 38, n. 8, p. 1627-1635, 2009.

GODOY, M. M.; ALVES, J. B.; MONTEIRO, A. L. G.; VALÉRIO FILHO, W. V. Parâmetros reprodutivo e metabólico de vacas da raça Guzerá suplementadas no pré e pós-parto. Revista Brasileira de Zootecnia, Viçosa, v. 33, n. 1, p. 103-111, 2004.

LAKE, S. L.; SCHOLLJEGERDES, E. J.; ATKINSON, R. L.; NAYIGIHUGU, V.; PAISLEY, S. I.; RULE, D. C.; MOSS, G. E.; ROBINSON, T. J.; HESS, B. W. Body condition score at parturition and postpartum supplemental fat effects on cow and calf performance. Journal of Animal Science, Champaign, v. 83, p. 29082917, 2005.

LAMB, G. C.; STEVENSON, J. S.; KESLER, D. J. H. A.; GARVERICK, D. R.; BROWN, B. R.; SALFEN, D. E. Inclusion of an intravaginal progesterone insert plus GnRH and prostaglandin F2 $\alpha$ for ovulation control in postpartum suckled beef cows. Journal of Animal Science, Champaign, v. 79, p. 2253-2259, 2001.

MALVEN, F. V. Pathophysiology of the puerperium: definition of the problem. In: INTERNATIONAL CONGRESS AN ANIMAL REPRODUCTION AND ARTIFICIAL INSEMINATION, 10, Urbana, 1984. Conference... Urbana: University of Illinois at UrbanaChampaign, 1984. p. 10-14.

MARQUES, M. O.; REIS, E. L.; CAMPOS FILHO, E. P.; BARUSELLI, P. S. Efeitos da administração de eCG e de benzoato de estradiol para sincronização da ovulação em vacas Bos taurus taurus $\mathrm{x}$ Bos taurus indicus no período pós-parto. In: SIMPÓSIO INTERNACIONAL DE REPRODUCCIÓN ANIMAL, 5., 2003, Huerta Grande. Anais... Huerta Grande: [s.n], 2003. v. 1, p. 392.

MCMANUS, C.; SAUERESSIG, M. G.; FALCÃO, R. A.; SERRANO, G.; MARCELINO, K. R. A.; PALUDO, G. R. Componentes reprodutivos e produtivos no rebanho de corte da embrapa cerrados. Revista Brasileira de Zootecnia, Viçosa, v. 31, n. 2, p. 648-657, 2002.

MENEGHETTI, M.; VASCONCELOS, J. L. M. Mês de parição, condição corporal e resposta ao protocolo de inseminação artificial em tempo fixo em vacas de corte primíparas. Arquivo Brasileiro de Medicina Veterinária e Zootecnia, Belo Horizonte, v. 60, n. 4, p. 786-793, 2008.

MENEGHETTI, M.; LOSI, T. C.; MARTINS JUNIOR, A. P. Uso de protocolo de IATF associado a diagnóstico precoce de gestação e ressincronização como estratégia para maximizar o número de vacas gestantes por IA em estação de monta reduzida. A Hora Veterinária, Porto Alegre, v. 147, p. 25-27, 2005.

MORRISON, D. G.; SPITZER, J. C.; PERKINS, J. L. Influence of prepartum body condition score change on reproduction in multiparous beef cows calving in moderate body condition. Journal of Animal Science, Champaign, v. 77, n. 5, p. 1048-1054, 1999.

PFEIFER, L. F. M.; VARELA, A. S.; FONTOURA JUNIOR, J. A. S.; SCHNEIDER, A.; CORREA, M. 
N.; DIONELLO, N. J. L. Efeito da condição corporal avaliada no diagnóstico de gestação sobre o momento da concepção e taxa de prenhez em vacas de corte. Acta Scientiae Veterinariae, Porto Alegre, v. 35, n. 3, p. 303307, 2007.

RICHARDS, M. W.; SPITZER, J. C.; WARNER, M. B. Effect of varying levels of postpartum nutrition and body condition at calving on subsequent reproductive performance in beef cattle. Journal of Animal Science, Champaign, v. 62, p. 300-306, 1986.

RIGOLON, L. P.; PRADO, I. N.; CAVALIERI, F. L. B.; NEGRÃ̃, J. A.; SILVA, R. R.; MARQUES, J. A. Níveis de ingestão de matéria seca sobre metabólitos e hormônios circulantes e hormônios foliculares em novilhas de corte. Revista Brasileira de Saúde e Produção Animal, Salvador, v. 9, n. 2, p. 367-383, 2008.

RUAS, J. R. M.; BRANDÃO, F. Z.; SILVA FILHO, J. M.; BORGES, A. M.; PALHARES, M. S.; CARVALHO, B. C.; BORGES, L. E. Indução do estro no pós-parto em vacas primíparas Holandês-Zebu. Arquivo Brasileiro de Medicina Veterinária e Zootecnia, Belo Horizonte, v. 57, n. 4, p. 476-484, 2005.

SAMPEDRO, D.; GALLI, I.; VOGEL, O. Condición corporal: uma herramienta para planificar el manejo del rodeo de cria. Mercedes: Ediciones INTA, 2003. 30 p. (Serie técnica, 30).

SANTOS, S. A.; ABREU, U. G. P.; SOUZA, G. S.; CATTO, J. B. Condição corporal, variação de peso e desempenho reprodutivo de vacas de cria em pastagem nativa no Pantanal. Revista Brasileira de Zootecnia, Viçosa, v. 38, n. 2, p. 354-360, 2009.

SHORT, R. E.; BELLOWS, R. H.; STAIGMILLER, R. B.; BERARDINELLI, J. G.; CUSTER, E. E. Physiological mechanisms controlling anestrus and infertility in postpartum beef cattle. Journal of Animal Science, Champaign, v. 68, p. 799-816, 1990.
SONOHATA, M. M.; OLIVEIRA, C. A. L.; CANUTO, N. G. D.; ABREU, U. G. P.; FERNANDES, D. D. Escore de condição corporal e desempenho reprodutivo de vacas no Pantanal do Mato Grosso do Sul - Brasil. Revista Brasileira de Saúde e Produção Animal, Salvador, v. 10, n. 4, p. 988-998, 2009.

TESS, M. W.; KOLSTAD, B. W. Simulation of cow-calf production system in a range environment: II. Model development. Journal of Animal Science, Champaign, v. 78, n. 5, p. 1159-1169, 2000.

VALLE, E. R.; ANDREOTTI, R.; THIAGO, L. R. L. de S. Estratégias para aumento da eficiência reprodutiva e produtiva em bovinos de corte. Campo Grande: EMBRAPA-CNPGC, 1998. 80 p. (EMBRAPA-CNPGC. Documentos, 71).

VASCONCELOS, J. L. M.; VILELA, E. R.; SÁ FILHO, O. G. Remoção temporária de bezerros em dois momentos do protocolo de sincronização da ovulação GnRH-PGF2 $\alpha$-BE em vacas Nelore pós-parto. Arquivo Brasileiro de Medicina Veterinária e Zootecnia, Belo Horizonte, v. 61, n. 1, p. 95-103, 2009.

VIEIRA, A.; JOSÉ LOBATO, F. P.; TORRES JUNIOR, R. A. A.; CEZAR, I. M.; ORREA, E. S. Fatores Determinantes do desempenho reprodutivo de vacas nelore na região dos cerrados do Brasil Central. Revista Brasileira de Zootecnia, Viçosa, v. 34, n. 6, p. 24082416, 2005. Suplemento.

WETTEMANN, R. P.; BOSSIS, I. Energy intake regulates ovarian function in beef cattle. Indianápolis: American Society of Animal Science, Champaign, 1999. $10 \mathrm{p}$.

WHISNANT, C. S.; KISER, T. E.; THOMPSON, F. N. Opioid inhibition of luteinizing hormone secretion during the postpartum period in suckled beef cows. Journal of Animal Science, Champaign, v. 63, n. 5, p. 1445-1448, 1986. 